\title{
Implementation of Energy Efficient Integer Wavelet Transform in Spartan 3E FPGA
}

\author{
D.Vijendra Babu \\ Asst.Proffesor \&Head, Department of \\ Electronics and Communication Engineering, \\ Aarupadai Veedu Institute of Technology, \\ Vinayaka Missions University, Chennai, India
}

\author{
Dr.N.R.Alamelu \\ Principal, Aarupadai Veedu Institute of \\ Technology, Vinayaka Missions University, \\ Chennai, India
}

\begin{abstract}
In this paper, we present an evaluation of various wavelet filters in the context of an energy efficient integer wavelet transform on SPARTAN 3E FPGA. The usage of different wavelet filters (Daubechies-9/7, Daubechies-5/3, Haar, etc.) for differential frames is investigated, where we mainly focus on computation time and average image quality of the video stream. We port this software system to a hardware system. Based on wavelet transform, we propose an Energy Efficient Wavelet Image Transform Algorithm (EEIWTA) for lossy compression of video, enabling significant reductions in computation as well as communication energy needed, with minimal degradation in image quality. Additionally, we identify video compression parameters that can be used to effect trade-offs between the energy savings, quality of the image and required communication bandwidth. Finally, the proposed system was implemented. We present energy efficient, adaptive data codec for image and video that can significantly minimize the energy required for wireless image communication, while meeting bandwidth constraints of the wireless networks, as the image quality and latency constraints of the wireless service. In this paper, the optimum method of wavelet transformation is explored. Performance Measure of different Wavelets is compared with and without elimination scheme. Simulation results show the important properties of wavelet, which have to be considered for, image compression. Peak signal to noise ratio (PSNR) is used as a measure to compare wavelet filters. By using these wavelets and compression, we can achieve an optimum balance between the performance metrics like PSNR and Compression Ratio and reduces the Mean Square Error. Our results provide better results in terms of computation time and PSNR ratio.
\end{abstract}

\section{Keywords \\ Component,DWT,EEWTA,Spartan 3E FPGA}

\section{INTRODUCTION}

During the past decades, with the birth of wavelet theory and multiresolution analysis, wavelet based image compression techniques have been extensively studied and tremendously improved .An overview of wavelets has brought to the fields as diverse as video conferencing, remote sensing and biomedical imaging and computer graphics or turbulence, is given in ${ }^{[1]}$.From a historical point of view, Joseph Fourier in the nineteenth century laid the foundation of wavelet analysis with his theory of frequency analysis, which proved to be enormously powerful and important[2]. The first citation of "Wavelet" seems to be in 1909, by
Alfred Haar. In the late nineteen-eighties, Daubechies, Mallat and Meyer explored and applied the ideas of wavelet transforms. There was a great amount of literature addressing the wavelet based signal processing techniques such as compression.

\section{Overview of wavelet transform}

The wavelet-based transform uses a 1-D sub band decomposition process in which a 1-D set of sample is converted into the low-pass sub band (Li) and high-pass sub band (Hi). Where i represents level of decomposition. The low-pass sub band represents a down sampled low-resolution version of the original image. The highpass sub band represents residual information of the original image. In 2-D sub band decomposition, the entire process is carried out by executing 1-D sub band decomposition twice, first in one direction (horizontal), then in the orthogonal (vertical) direction. For example, the low-pass sub band (Li) resulting from the horizontal direction is further decomposed in the vertical direction, leading to LLi and LHi sub bands. Similarly, the high pass sub band (Hi) is further decomposed into HLi and HHi. After one level of transform, the image can be further decomposed by applying the 2-D subband decomposition to the existing LLi subband. This iterative process results in multiple "transform levels". We refer to the subband LLi as a low-resolution subband and high-pass sub bands LHi, HLi, HHi as horizontal, vertical, and diagonal subband respectively since they represent the horizontal, vertical and diagonal residual information of the original image.

The Discrete Wavelet Transform (DWT) is a versatile signal processing tool that finds many engineering and scientific applications. One area in which the DWT has been particularly successful is in image compression and it has been adopted in the upcoming JPEG2000 image compression standard. Recently the concept of lifting has thrown net insight and ideas on wavelets and has served to enhance the power and versatility of wavelet transforms. Lifting provides an efficient way to implement the DWT and the computational efficiency of the lifting implementation can be up to $100 \%$ higher than the traditional direct convolution based implementation. The lifting approach is adopted in JPEG2000. The lifting scheme has also provided an easy way to construct new types of wavelet transform, which can be nonlinear. 


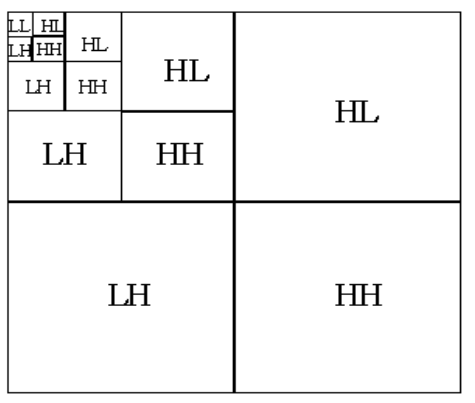

Figure 1. Wavelet Decomposition

\section{$A$. Integer Wavelet Transform}

The Integer Wavelet Transforms (IWT) maps integers to integers and allows for perfect invertibility with finite precision arithmetic. A simple and effective way to construct IWT is to first factor the traditional DWT into lifting steps and then to apply a rounding operation at each step. The IWT can thus be used for lossless compression of medical images. One of the main advantages of using the wavelet transform for compression is that it provides a multiresolution representation of the image, which other techniques like spatial-domain prediction cannot offer. The multiresolution representation allows the transmission of the lower resolution version of the image first, followed by transmission of successive. This mode of transmission is useful when the bandwidth is limited and the image sizes are large, e.g. 2D and 3D medical images for telemedicine applications. The transmission can be stopped at the client end if it is deemed that the received image at the current resolution is sufficient or the image is not of interest at the user end. However, a full resolution lossless version of the image can be received if so desired. Note that the IWT can also be used for lossy compression and it has certain advantages over the traditional DWT. The IWT can be used in a unified lossy and lossless codec [5] and a seamless transition between virtually lossless and strictly lossless can be achieved. The IWT also have the potential for simpler implementation as many of the operands are integer and hence widely used in compression systems in industries [1].

\section{II. proposed wavelet}

\section{A. Energy Efficient Integer Wavelet}

The proposed video codec works on the algorithm based on EEWTA (Dong-Gi Lee 2002) [1]. The EEWTA follows the classical paradigm of transformation, quantization and encoding but exploits the multiresolution property of wavelets. A modified wavelet transformation is employed during the image decomposition process.

A wavelet-based transform algorithm (EEWTA) that aims at minimizing computation energy (by reducing the number of arithmetic operations and memory accesses) and communication energy (by reducing the number of transmitted bits) is undertaken. Further, the algorithm aims at effecting energy savings while minimally influencing the quality of the image. EEWTA exploits the numerical distribution of the high-pass coefficients to eliminate a large number of samples from consideration in the image compression process.
The high-pass coefficients are generally represented by small integer values. Because of the numerical distribution of the high-pass coefficients and the effect of the quantization step on small valued coefficients, we can estimate the high-pass coefficients to be zeros (avoids computing them) and incur minimal image quality loss.

This approach has two main advantages. First, because the high-pass coefficients do not have to be computed, EEWTA helps to reduce the computation energy consumed during the wavelet image compression process by reducing the number of executed operations. Second, because the encoder and decoder are aware of the estimation technique, no information needs to be transmitted across the wireless channel, thereby reducing the communication energy required.

During the wavelet transform, each input image goes through the row and column transform decomposing the image into four sub bands (LL, LH, HL and HH). The modified wavelet transformation is used for EEWTA to implement the $\mathrm{HH}$ elimination and $\mathrm{H}^{*}$ elimination methods. To implement the $\mathrm{HH}$ elimination method after the row transform, the high pass coefficients are only fed into the low pass filter and not the high pass filter in the following column transform step as shown in Fig. 4.1. This avoids the generation of the diagonal subband (HH). This method saves computational energy as in accordance with the analysis of computation that denotes the two loads namely data access load and computation load that are associated with the subband generation

\section{HH elimination method}

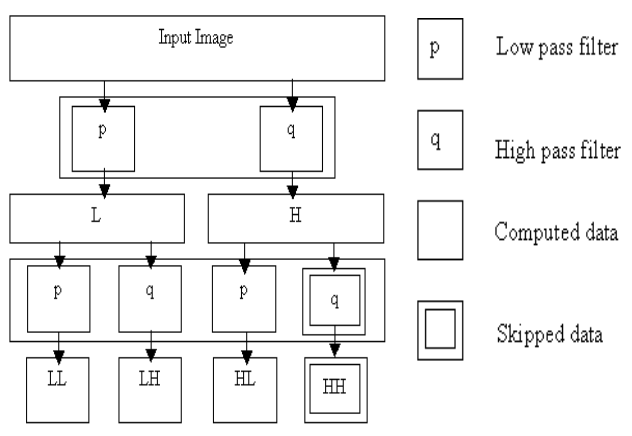

Figure 2. HH elimination method of EEIWT

The HH elimination method, which eliminates the insignificant high pass subband, can then be then be continued to process the image by a greater depth of transformation. The multiple level of decomposition is performed in the same way neglecting the high pass subband at the first level of decomposition and be subjected to a normal two-dimensional wavelet transform from the second level with the consideration of the image loss.

A number of useful information is lost when the elimination is continued after one or two levels of decomposition. To retain the image quality, the elimination level is performed only in the lower levels and to achieve high compression and to save computational energy, elimination level is performed to high levels of transformation. 


\section{A. H* Elimination Method}

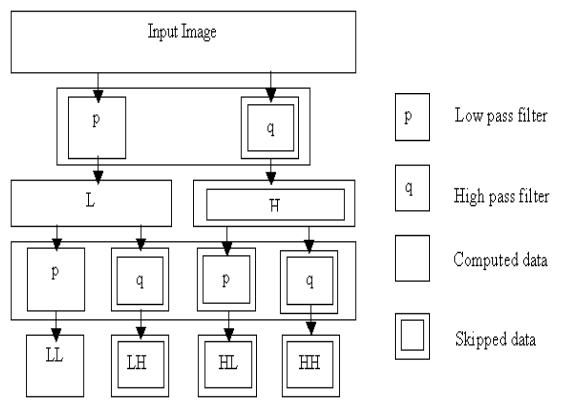

Figure 3. $\mathrm{H}^{*}$ elimination method of EEIWT

The $\mathrm{H}^{*}$ elimination method also employs the modified wavelet transformation and in this method it retains the most significant low pass subband and eliminates all the high pass sub bands. i.e., horizontal, vertical and diagonal sub bands. In this method, the input image is processed only through the low pass filter during both the row and column transform steps. The higher levels of image decomposition can be carried out in the same way, eliminating all high pass sub bands only if the compression is of major concern and with image quality being of importance, the elimination process can be stopped at the lower levels of transform.

The proposed video codec employs the EEWTA algorithm and its analysis of computational and data access loads (Dong-Gi Lee 2002) for energy savings.

\section{Implementation Results}

The hardware system consists of a Spartan 3e FPGA starter kit from Digilent inc.Raw pixel data is passed on to the FPGA through UART for Wavelet encoding and decoding. Data is then decomposed into sub bands and Wavelet transform (WT) is performed. The wavelet engine can perform up to 6 wavelet decomposition levels on a tile. The wavelet engine on Spartan FPGA supports a 9/7 irreversible (using fixed tables) wavelet transform and the $5 / 3$ wavelet transform in reversible and irreversible modes. At the end of the wavelet engine's pipeline, the computed wavelet coefficients are then written to internal memory and then stored in SDRAM.Wavelet encoded data is again ported to internal memory and to the main processing unit FPGA for wavelet decoding. We concentrate on the computational effort, number of logic cells occupied and we used to compare the performance with $5 / 3$ filters and our proposed wavelet. In the results, the performance of using these filters is simulating in Mat Lab and EDK 10.1, comparison is show in terms of computation time and PSNR.

\section{Performance metrics}

Although the performance of a wavelet algorithm can be measured in a variety of ways, speed, storage and reconstructed image quality. Unfortunately, reconstructed image quality tends to be subjective and metrics that have been designed to approximate it do not always agree with a human viewer's perceptions. Having said that, however, there are some metrics that occur frequently as stand-ins for image quality: RMSE, MSE, PSNR, MAE, and others

\section{A. Image Quality}

Although there are several metrics that tend to be indicative of image quality, each of them has situations in which it fails to coincide with an observer's opinion. However, since running human trials is generally prohibitively expensive, a number of metrics are often computed to help judge image quality. The metrics that see the widest usage are generally quite simple to compute. Once a metric has been used in a seminal article that presents test results; other researchers will continue using that metric so that their data can be compared to the previous work. This last reason was the determining factor in the use of peak signal-to-noise ratio (PSNR).

\section{B. MSE, RMSE}

Two other quantities that appear frequently when comparing original and reconstructed or approximated data are (root) mean square error. These measures will not be seriously skewed by a single anomaly, since they are measuring average behavior. RMSE produces the same units as the original image data, so its results are easy to interpret. Again it should be remembered that these metrics attempt to measure an inverse to image quality. Where $\mathrm{m}$ ,$n$ represents the rows and columns of the image.X(i,j) represents input image and $\mathrm{Y}(\mathrm{i}, \mathrm{j})$ represents Output image. MSE $=1$ MSEMSE

$$
\begin{aligned}
& M S E=\frac{1}{m n} \sum_{t, 5}\left(x_{i g}-y_{b j}\right)^{2} \\
& R M S E=\sqrt{\frac{1}{m n} \sum_{t, 5}\left(x_{i g}-y_{b j}\right)^{2}}
\end{aligned}
$$

\section{PSNR}

Peak signal-to-noise ratio has two definitions, the original more precise definition, and the second easier to compute and more commonly used. It is this second definition that we use throughout this report. This is the first metric mentioned so far where the results generally run proportional to image quality rather than the inverse.

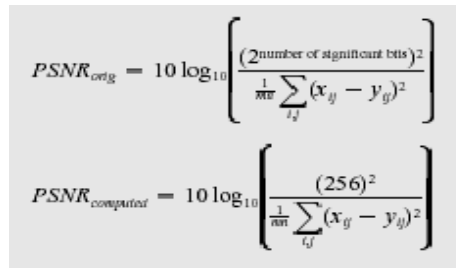

\section{Simulation results}

In our experiment, we have analyzed the Daubechies 5/3 Wavelet with our adaptive EEWITA Cameraman (256x256) and Lena (512 $\mathrm{x} 512)$ image has been used. These test images are of different frequency contents, different size and different resolution.

Firstly we have simulated the computation time in Mat Lab 7.05 and the results are shown in table. 
Table I Computation time for NO elimination, $\mathrm{HH}$ and

$\mathrm{H}^{*}$

Profile Summary

Generated 20-Aug-2009 05:32:15 using cpu time.

\begin{tabular}{|c|c|c|c|c|}
\hline Function Name & $\underline{\text { Calls }}$ & Total Time & $\underline{\text { Self Time }}^{*}$ & $\begin{array}{l}\text { Total Time Plot } \\
\text { (dark band = self time) }\end{array}$ \\
\hline dbno & 1 & $0.247 \mathrm{~s}$ & $0.247 \mathrm{~s}$ & \\
\hline dbhh & 1 & $0.231 \mathrm{~s}$ & $0.231 \mathrm{~s}$ & \\
\hline dbh & 1 & $0.154 \mathrm{~s}$ & $0.154 \mathrm{~s}$ & \\
\hline
\end{tabular}

Self time is the time spent in a function excluding the time spent in its child functions. Self time also includes overhead resulting from the process of profiling.

Secondly, we used Xilinx platform studio 10.1 for evaluating the logical resources of the FPGA.We used the following architecture to verify the Logic utilization and Logic Distribution. The table shows our proposed wavelet requires only less logic utilization.

$\begin{array}{ll}\text { Architecture } & \text { : SPARTAN3E } \\ \text { Device } & : \text { XC3S500E } \\ \text { Package } & : \text { FG320 } \\ \text { Speed Grade } & :-4\end{array}$

Table I Logic Utilization and Logic Distribution in Spartan 3E

\begin{tabular}{|c|c|c|c|c|}
\hline \multicolumn{5}{|c|}{ Logic Utilization } \\
\hline $\begin{array}{c}\text { TAAR } \\
\text { Registers }\end{array}$ & $52 \%$ & $40 \%$ & $32 \%$ & $27 \%$ \\
\hline $\begin{array}{c}\text { Total Number of Flip } \\
\text { Flops }\end{array}$ & 3,902 & 3,020 & 2,721 & 2,533 \\
\hline $\begin{array}{c}\text { Number used as } \\
\text { Latches }\end{array}$ & 1 & 1 & 1 & 1 \\
\hline $\begin{array}{c}\text { Number of 4 input } \\
\text { LUTs }\end{array}$ & $51 \%$ & $39 \%$ & $32 \%$ & $26 \%$ \\
\hline $\begin{array}{c}\text { Number of occupied } \\
\text { Slices }\end{array}$ & $72 \%$ & $64 \%$ & $55 \%$ & $55 \%$ \\
\hline Number used as logic & 4,020 & 2,723 & 2,200 & 2,060 \\
\hline Number of DCMs & $50 \%$ & $50 \%$ & $50 \%$ & $50 \%$ \\
\hline $\begin{array}{c}\text { Number of } \\
\text { BUFGMUXs }\end{array}$ & $40 \%$ & $23 \%$ & $23 \%$ & $20 \%$ \\
\hline Number of RAMB16s & $49 \%$ & $42 \%$ & $38 \%$ & $35 \%$ \\
\hline $\begin{array}{c}\text { Number of } \\
\text { MULT18X18SIO }\end{array}$ & $25 \%$ & $20 \%$ & $16 \%$ & $15 \%$ \\
\hline \multicolumn{2}{|c|}{ Logic Distribution } \\
\hline
\end{tabular}

Here we have given the experimental results for Wavelets, which provide better PSNR in corresponding Wavelets. The comparison of Wavelet families provides better compression performance for test as shown in figure. We are also presenting results of test images in terms of visual quality are shown in figure.
Table II Comparison of PSNR for HAAR, NO, HH, H*

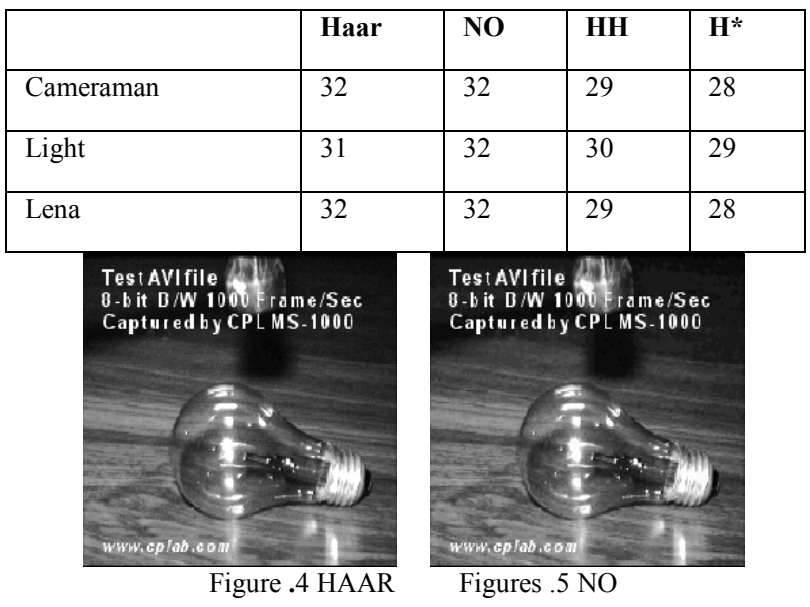

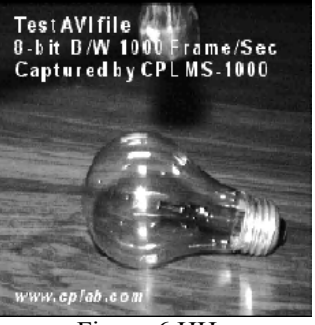

Figure 6.HH

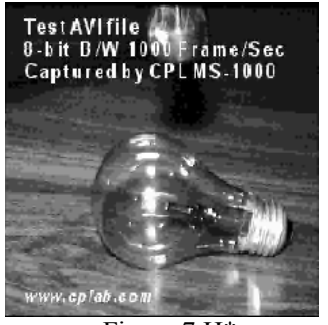

Figure 7.H*

\section{CONCLUSION}

This paper presented an analysis and comparison of the Wavelet families with our proposed wavelet using for image compression considering PSNR, Computation time and visual quality of image as quality measure. A comparative study of various Wavelet families has done and the effects of Daubechies and our proposed Wavelet have been tested, the PSNR, computation time, logic utilization and logic distribution in Spartan 3E FPGAs also observed. We analyzed the results for a wide range of Wavelet families and found that our HH elimination method and $\mathrm{H}^{*}$ Ellimination method provides less computation time and uses less logical resources in FPGA.The Compression performance of image depends not only on the size of the image but also on the content and resolution of the image, so when we used our proposed wavelet in an image compression scheme and we got affair image quality and better compression ratio. As future work these Wavelet can be extended to 3D Wavelets and check the performance of the codec by implemented in any one of the image and video compression scheme. 


\section{REFERENCES}

[1] Hongyang Chao, Howard P. Fisher, Paul S. Fisher (2005), 'Image compression using an integer reversible wavelet transform with a property'

[2] Uli Grasemann and Risto Miikkulainen (2005), 'Effective Image Compression using Evolved Wavelets.' In Proceedings of the Genetic and Evolutionary Computation Conference.

[3] Antonini. M, Barlaud. M, Mathieu. P, and Daubechies. I (1992), 'Image coding using wavelet transform', IEEE Trans. on Image Processing, Vol. 1, no. 2, pp 205-220.

[4] Margaret A. Lepley (1997), 'AWIC: Adaptive Wavelet Image Compression for still image', MTR97B0000040, The MITRE Corporation, Bedford, MA, September.
[5] Rushanan. J.J (1997), 'AWIC-Adaptive Wavelet Image Compression for Still Image', MTR97B0000041, the MITRE Corporation, Bedford,

MA.

[6] Sayood. K (2000), 'Introduction to Data Compression', San Mateo, CA-Mogan Kaufmann.

[7] Shapiro, J.M (1993), 'Embedded image coding using zero trees of wavelet coefficients', IEEE Transactions on Signal Processing, Vol.41, No.12, p.3445-3462. 